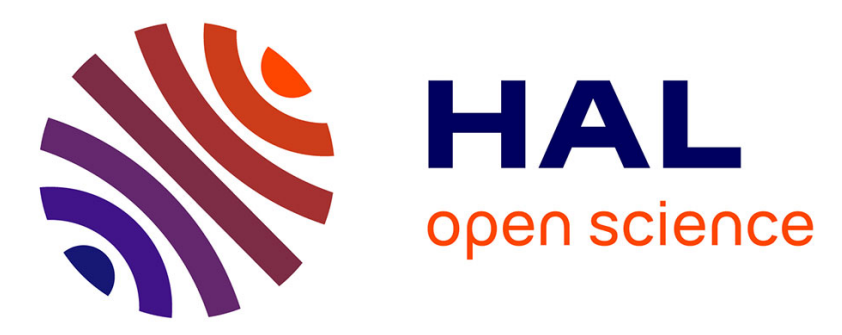

\title{
Finding common weights based on the DM's preference information
}

\author{
G.R. Jahanshahloo, M Zohrehbandian, A Alinezhad, S Abbasian Naghneh, H \\ Abbasian, R Kiani Mavi
}

\section{> To cite this version:}

G.R. Jahanshahloo, M Zohrehbandian, A Alinezhad, S Abbasian Naghneh, H Abbasian, et al.. Finding common weights based on the DM's preference information. Journal of the Operational Research Society, 2011, 10.1057/jors.2010.156 . hal-03212809

\section{HAL Id: hal-03212809 \\ https://hal.science/hal-03212809}

Submitted on 30 Apr 2021

HAL is a multi-disciplinary open access archive for the deposit and dissemination of scientific research documents, whether they are published or not. The documents may come from teaching and research institutions in France or abroad, or from public or private research centers.
L'archive ouverte pluridisciplinaire HAL, est destinée au dépôt et à la diffusion de documents scientifiques de niveau recherche, publiés ou non, émanant des établissements d'enseignement et de recherche français ou étrangers, des laboratoires publics ou privés. 


\title{
Finding common weights based on the DM's preference information
}

\author{
G.R. Jahanshahloo ${ }^{a}$, M. Zohrehbandian ${ }^{b, *, \dagger}$ A. Alinezhad ${ }^{c}$, \\ S. Abbasian Naghneh ${ }^{b}$, H. Abbasian ${ }^{b}$, R. Kiani Mavi ${ }^{d}$ \\ ${ }^{a}$ Department of Mathematics, Science \& Research Branch, Islamic Azad University, Tehran, Iran. \\ ${ }^{b}$ Department of Mathematics, Karaj Branch, Islamic Azad University, P.O.Box 31485-313, Karaj, Iran. \\ ${ }^{c}$ Department of Industrial Engineering, Qazvin Branch, Islamic Azad University, Qazvin, Iran. \\ ${ }^{d}$ Department of Industrial Management, Qazvin Branch, Islamic Azad University, Qazvin, Iran.
}

\begin{abstract}
Data Envelopment Analysis (DEA) is basically a linear programming based technique used for measuring the relative performance of organizational units, referred to as Decision Making Units (DMUs). The flexibility in selecting the weights in standard DEA models deters the comparison among DMUs on a common base. Moreover, these weights are not suitable to measure the preferences of a decision maker (DM). For dealing with the first difficulty, the concept of common weights was proposed in the DEA literature. But, none of the common weights approaches address the second difficulty. This paper proposes an alternative approach we term 'preference common weights' which is both practical and intellectually consistent with the DEA philosophy. To do this, we introduce an MOLP model in which objective functions are input/output variables subject to the constraints similar to the equations which define production possibility set (PPS) of standard DEA models. Then by using the Zionts-Wallenius method, we can generate common weights as the DM's underlying value structure about objective functions.
\end{abstract}

Keywords: Efficiency Analysis, Data Envelopment Analysis, Common Weights, Multiple Criteria Decision Making, Value Function.

\footnotetext{
${ }^{*}$ Corresponding Author. E-mail Address: zohrebandian@yahoo.com

${ }^{\dagger}$ This work is supported by Islamic Azad University-Karaj Branch, and the author is thankful to it.
} 


\section{Introduction}

Data Envelopment Analysis (DEA), introduced by Charnes et al. (1978), is a non-parametric extremal method for evaluation of the relative efficiency of a group of similar units, called Decision Making Units (DMUs). DEA gives a measure of efficiency, which is essentially defined as a ratio of weighted outputs to weighted inputs. Charnes et al.'s idea is to define the efficiency measure by assigning to each unit the most favorable weights as long as the efficiency scores of all DMUs calculated from the same set of weights, do not exceed one.

This flexibility in selecting the weights deters the comparison among DMUs on a common base. A possible answer to this difficulty lies in the specification of common weights, which was first introduced by Roll et al. (1991). Research about the idea of common weights has developed gradually in recent years. Some of the studies in this field are Roll and Golany (1993), Doyle and Green (1996), Jahanshahloo et al. (2005), Kao and Hung (2005), Karsak and Ahiska (2005), Zohrehbandian et al. (2010).

As an extension of the previous studies, this paper seeks to develop an interactive multiple objective linear programming (MOLP) model that incorporates preference structures for obtaining common weights in DEA. To achieve this goal, we introduce an MOLP model with objective functions as input/output variables subject to the constraints similar to the equations that define the production possibility set (PPS) of standard DEA models. Then by using the Zionts-Wallenius method, we can generate common weights as the DM's underlying value structure about objective functions. We term this approach 'preference common weights' which is both practical and intellectually consistent with the DEA philosophy.

For solving the proposed MOLP model by using the Zionts-Wallenius method, a DM is assumed to have only an implicit utility function of these objective functions and no explicit knowledge of the utility function that he wishes to maximize. The method uses an implicit function on an interactive basis and to resolve the conflicts inherent in the given multiple objectives, the DM is required only to provide answers to certain 'yes' or 'no' questions on feasible tradeoffs presented to him.

The plan for the rest of this paper is as follows. In section 2 we present a brief discussion about Zionts-Wallenius method in solving the MOLP problems. The mathematical foundation of our method for finding a common set of weights is discussed in Section 3. A numerical example is presented in section 4 and finally, section 5 draws the conclusive remarks. 


\section{MCDM Preliminaries}

Although the DEA and MCDM approaches are different regarding how efficiency is measured in practice, some of the authors have underlined the equivalence between the notion of efficiency in DEA and MCDM; e.g. Giokas (1997) and Golany (1988). Furthermore, several authors have pointed out some close connections between DEA and MCDM; see Belton and Vickers (1993), Bouyssou (1999), Estellita et al. (2004), Giokas (1997), Golany (1988), Joro et al. (1998), Stewart (1996), Xiao and Reeves (1999), Zhu (1996). Interestingly, Charnes and Cooper have also had a significant impact on the development of MCDM through the development of goal programming.

An MOLP problem is a case of MCDM problems, which can be written as follows:

$$
\begin{array}{cc}
\text { Max } & F(x)=\left\{f_{i}(x)=C_{i} x, i=1, \cdots, p\right\} \\
\text { s.t. } & x \in X=\left\{x \in R_{+}^{n} \mid a_{i} x=b_{i}, i=1, \cdots, m\right\}
\end{array}
$$

In order to solve the above problem (identifying the efficient solutions), there are many different methods in the literature. One of these methods is an interactive programming method proposed by Zionts and Wallenius (1976). In this method, it is assumed that the utility function $U$ is a linear function of the objective function variables $u_{i}=f_{i}(x), i=1, \cdots, p$, but the precise weights in such a function are not known explicitly. Below, we introduce the steps of this well known method.

Step 0: The Zionts-Wallenius method, first, chooses an arbitrary set of positive multipliers or weights, $\gamma_{i} \geq \varepsilon$ satisfying $\sum_{i=1}^{p} \gamma_{i}=1$, and generates a composite objective function. The composite objective function is then optimized to produce an extreme efficient solution $x^{*}$ to the problem.

Step 1: For each nonbasic variable $x_{l}$, compute the value of the $w_{i l} i=1, \cdots, p$, as

$$
w_{i l}=\frac{f_{i}\left(x^{*}\right)-f_{i}(\bar{x})}{\overline{x_{l}}},
$$

where $\bar{x}$ is an optimal solution of model (3).

$$
\begin{array}{cc}
\operatorname{Max} & x_{j} \\
\text { s.t. } & x \in X=\left\{x \in R_{+}^{n} \mid a_{i} x=b_{i}, i=1, \cdots, m\right\}
\end{array}
$$

Step 2: Solve model (4), where NBV is the set of nonbasic variables.

$$
\begin{array}{ll}
\text { Min } & \sum_{i=1}^{p} w_{i l} \gamma_{i} \\
\text { s.t. } & \sum_{i=1}^{p} w_{i j} \gamma_{i} \geq 0 \quad j \neq l, j \in N B V
\end{array}
$$




$$
\begin{aligned}
& \sum_{i=1}^{p} \gamma_{i}=1 \\
& \gamma_{i} \geq 0, \quad i=1, \cdots, p
\end{aligned}
$$

Test 1 If the optimal value of model (4) is negative, the variable $x_{l}$ is efficient,

Test 2 If the optimal value of model (4) is nonnegative, the variable $x_{l}$ is not efficient.

Step 3: For each efficient variable, the DM is asked: Here is a trade. Are you willing to accept a decrease in objective function $u_{1}$ of $w_{1 j}$, a decrease in objective function $u_{2}$ of $w_{2 j}, \cdots$, and a decrease in objective function $u_{p}$ of $w_{p j}$ ? Respond 'yes', 'no' or 'indifferent' to the trade.

If the responses are all no for all efficient variables, terminate the procedure and take $\gamma_{i}$ 's as the bast set of weights. Otherwise, using the DM's responses, we construct constraints to restrict the choice of the weights $\gamma_{i}$ to be used in finding a new efficient solution.

Step 4: For each 'yes' response construct an inequality of the form

$$
\sum_{i=1}^{p} w_{i j} \gamma_{i} \leq-\varepsilon
$$

For each 'no' response, construct an inequality of the form

$$
\sum_{i=1}^{p} w_{i j} \gamma_{i} \geq \varepsilon
$$

For each response of indifference, construct an equality of the form

$$
\sum_{i=1}^{p} w_{i j} \gamma_{i}=0 .
$$

A feasible solution to the following set of constraints is found:

All previously constructed constraints of the form (5), (6), (7) and

$$
\begin{gathered}
\sum_{i=1}^{p} \gamma_{i}=1, \\
\gamma_{i} \geq \varepsilon \quad i=1, \cdots, p .
\end{gathered}
$$

Step 5: The process is then repeated by the resulting set of $\gamma_{i}$ 's and optimization of composite objective function to produce a new extreme efficient solution to the problem. Go to step 1.

In this manner, convergence to an overall optimal solution with respect to the DM's implicit utility function is assured and finally, the overall optimal solution of $\gamma_{i}{ }^{\prime}$ s are the weights of objective functions with respect to the DM's implicit utility function. 


\section{Producing preference common weights}

Consider $\mathrm{n}$ production units, or DMUs, each of which consume varying amounts of $\mathrm{m}$ inputs in the production of s outputs. Suppose $\mathrm{x}_{i j} \geq 0$ denotes the amount consumed of the i-th input measure and $\mathrm{y}_{r j} \geq 0$ denotes the amount produced of the r-th output measure by the $\mathrm{j}$-th DMU. The PPS of obviously most widely used DEA model, CCR with constant returns to scale characteristic, is defined as semi-positive vectors $(\mathrm{x}, \mathrm{y})$ as follows:

$$
T_{c}=\left\{(x, y) \mid x \geq \sum_{j=1}^{n} \lambda_{j} x_{j}, \quad y \leq \sum_{j=1}^{n} \lambda_{j} y_{j}, \quad \lambda_{j} \geq 0 \quad j=1, \ldots, n\right\}
$$

Classical DEA models rely on the assumption that inputs have to be minimized and outputs have to be maximized. In other words, they evaluate DMUs and specify reference points due to this assumption. Here we notice that based on this assumption, a DEA model could be expressed as an MOLP problem applied to minimization of input variables and maximization of output variables subject to the constraints similar to the equations which define the PPS of standard DEA models. Hence, we propose following MOLP model which is intellectually consistent with the DEA philosophy:

$$
\begin{array}{cc}
\operatorname{Max} & -x_{1} \\
\vdots & \\
\operatorname{Max} & -x_{m} \\
\operatorname{Max} & y_{1} \\
\vdots & \\
\operatorname{Max} & y_{s} \\
\text { s.t. } & X \lambda \leq x \\
& Y \lambda \geq y \\
& \lambda \geq 0
\end{array}
$$

Like any MOLP model, the above model has no unique solution. But it is notable that its efficient solutions are defined analogously to the efficient frontier of CCR model. Now and due to the objective functions of this model, if we solve it by Zionts-Wallenius method, we can specify a proper set of preference weights that reflect the relative degree of DM's underlying value structure about inputs and outputs. In other words, we produce a preference common weights and then efficiency score of $\mathrm{DMU}_{j}, \mathrm{j}=1, \ldots, \mathrm{n}$, can be obtained by using these common weights as $\frac{\sum_{r=1}^{s} u_{r}^{*} y_{r j}}{\sum_{i=1}^{m} v_{i}^{*} x_{i j}}$. 
Roll et al. (1991) indicate that a general requirement for the common set of weights is that at least one DMU must attain efficiency 1 with the common weights. If there is no DMU with efficiency score 1 , then it is obvious that the efficiencies are under-estimated in the sense of relative comparison. In this sense and based on the following theorem, the efficiency scores obtained from our proposed method are not under-estimated and satisfied the general requirement.

Theorem There is a $\mathrm{DMU}_{p}, \mathrm{p} \in\{1, \ldots, n\}$ for which we have $\frac{\sum_{r=1}^{s} u_{r}^{*} y_{r p}}{\sum_{i=1}^{m} v_{i}^{*} x_{i p}}=1$.

Proof Suppose that we generate a composite objective function using $\left(u^{*}, v^{*}\right)$ multipliers and subject to the same constraints of (9). Then based on the duality, optimal objective value of this model is equal to zero. Hence, for all feasible solutions $(\lambda, x, y)$ of the model we have: $\sum_{r=1}^{s} u_{r}^{*} y_{r}-\sum_{i=1}^{m} v_{i}^{*} x_{i} \leq 0$, and there is an optimal solution $\left(\lambda^{*}, x^{*}, y^{*}\right)$ of the model for which we have: $\sum_{r=1}^{s} u_{r}^{*} y_{r}^{*}-\sum_{i=1}^{m} v_{i}^{*} x_{i}^{*}=0$. In other words, the equation of the form $\sum_{r=1}^{s} u_{r}^{*} y_{r}-\sum_{i=1}^{m} v_{i}^{*} x_{i}=0$ defines a supporting hyperplane that contains PPS in only one of the halfspaces and support it at virtual DMU $\left(x^{*}, y^{*}\right)$. But such a supporting hyperplane must support PPS at an observed DMU; e.g. $\mathrm{DMU}_{p}$. Therefore, we have: $\sum_{r=1}^{s} u_{r}^{*} y_{r p}-\sum_{i=1}^{m} v_{i}^{*} x_{i p}=0$, or $\frac{\sum_{r=1}^{s} u_{r}^{*} y_{r p}}{\sum_{i=1}^{m} v_{i}^{*} x_{i p}}=1$

Note that by slight manipulation of the proposed model (e.g. adding the constraint for sum of $\lambda_{j}$ ), we can develop the concept of producing a preference common weights to other DEA models.

\section{Numerical Example}

To illustrate the idea of the proposed approach, an example is utilized with 25 DMUs. Where each DMU uses 4 inputs to produce 3 outputs. Table 1 shows the value of these inputs and outputs.

The results of using the presented approach in section 3 for obtaining a preference common weights in Variable Returns to Scale (VRS) context is as follows:

Iteration 1 We first choose an arbitrary set of weights $\gamma=(0.143,0.143,0.143,0.143,0.143,0.143$, 0.143). The composite objective function was then optimized which produced $\left(x_{1}, x_{2}, x_{3}, x_{4}, y_{1}, y_{2}, y_{3}\right)$ $=(4236,3145,3334,4504,8423,9821,8821)$ with $\lambda_{4}=1$ as an extreme efficient solution to the problem. The set of nonbasic variables were $\lambda_{i}, \quad i=1, \cdots, 25, i \neq 4$ and solving model (2), by maximization of the nonbasic variable $\lambda_{i}, \quad i=1, \cdots, 25, i \neq 4$, caused to the optimal solution $\left(x_{i 1}, x_{i 2}, x_{i 3}, x_{i 4}, y_{i 1}, y_{i 2}, y_{i 3}\right)$ with optimal value equals to 1 . Determination of efficient variables were based on the estimation of $w_{i j}$ values which were introduced in table (2).

For each nonbasic variable $\lambda_{i}, i=1, \cdots, 25, i \neq 4$, model (4) was solved and variables $\lambda_{1}, \lambda_{6}, \lambda_{9}, \lambda_{10}, \lambda_{11}, \lambda_{15}, \lambda_{16}, \lambda_{17}, \lambda_{18}, \lambda_{19}, \lambda_{22}, \lambda_{23}, \lambda_{25}$, were determined as efficient variables. Then 


\begin{tabular}{|l|cccc|ccc|c|}
\hline $\mathrm{DMUs}_{\mathrm{s}}$ & $\mathrm{I}_{1}$ & $\mathrm{I}_{2}$ & $\mathrm{I}_{3}$ & $\mathrm{I}_{4}$ & $\mathrm{O}_{1}$ & $\mathrm{O}_{2}$ & $\mathrm{O}_{3}$ & Efficiency \\
\hline $\mathrm{DMU}_{1}$ & 3422 & 4012 & 4353 & 3525 & 8921 & 5842 & 7512 & 1.05942 \\
\hline $\mathrm{DMU}_{2}$ & 3899 & 4316 & 4528 & 4656 & 5618 & 7343 & 6200 & 1.43594 \\
\hline $\mathrm{DMU}_{3}$ & 3478 & 4802 & 3874 & 3270 & 5468 & 5698 & 5102 & 1.40834 \\
\hline $\mathrm{DMU}_{4}$ & 4236 & 3145 & 3334 & 4504 & 8423 & 9821 & 8821 & 1.12505 \\
\hline $\mathrm{DMU}_{5}$ & 4821 & 3910 & 4140 & 4756 & 9181 & 6879 & 7305 & 1.40201 \\
\hline $\mathrm{DMU}_{6}$ & 4110 & 3487 & 3546 & 3123 & 6752 & 6521 & 9700 & 1.08162 \\
\hline $\mathrm{DMU}_{7}$ & 3980 & 4512 & 3487 & 3676 & 8315 & 8400 & 7546 & 1.18137 \\
\hline $\mathrm{DMU}_{8}$ & 4741 & 4231 & 4123 & 4523 & 6458 & 5600 & 9000 & 1.37730 \\
\hline $\mathrm{DMU}_{9}$ & 3422 & 3568 & 3961 & 3999 & 8010 & 5000 & 5887 & 1.22153 \\
\hline $\mathrm{DMU}_{10}$ & 4802 & 3154 & 4215 & 3792 & 7039 & 6015 & 5642 & 1.56621 \\
\hline $\mathrm{DMU}_{11}$ & 3050 & 4988 & 3971 & 4823 & 9253 & 8433 & 5897 & 1.14583 \\
\hline $\mathrm{DMU}_{12}$ & 3645 & 3753 & 4270 & 4219 & 5812 & 4999 & 6658 & 1.31994 \\
\hline $\mathrm{DMU}_{13}$ & 4910 & 3999 & 4190 & 3190 & 7314 & 5488 & 4599 & 1.70783 \\
\hline $\mathrm{DMU}_{14}$ & 4720 & 3491 & 3564 & 4802 & 6541 & 8324 & 7895 & 1.39392 \\
\hline $\mathrm{DMU}_{15}$ & 3879 & 4258 & 3500 & 3613 & 8741 & 9541 & 7291 & 1.13478 \\
\hline $\mathrm{DMU}_{16}$ & 4512 & 4908 & 4208 & 3692 & 9718 & 9291 & 8102 & 1.21120 \\
\hline $\mathrm{DMU}_{17}$ & 3691 & 4325 & 3222 & 5000 & 5642 & 7518 & 9941 & 1.12987 \\
\hline $\mathrm{DMU}_{18}$ & 4321 & 3867 & 3224 & 4003 & 10000 & 6465 & 9429 & 1.10821 \\
\hline $\mathrm{DMU}_{19}$ & 3784 & 3312 & 3989 & 3722 & 9758 & 6128 & 6709 & 1.14007 \\
\hline $\mathrm{DMU}_{20}$ & 3465 & 4657 & 3874 & 4918 & 7302 & 7312 & 7032 & 1.23246 \\
\hline $\mathrm{DMU}_{21}$ & 4410 & 4415 & 4632 & 3558 & 8821 & 6218 & 8245 & 1.22949 \\
\hline $\mathrm{DMU}_{22}$ & 3333 & 3720 & 4228 & 3292 & 5912 & 7324 & 8914 & 1.00000 \\
\hline $\mathrm{DMU}_{23}$ & 3784 & 4666 & 4220 & 4818 & 7543 & 8499 & 9214 & 1.12713 \\
\hline $\mathrm{DMU}_{24}$ & 4825 & 4777 & 3890 & 4391 & 6100 & 7666 & 4521 & 1.82717 \\
\hline $\mathrm{DMU}_{25}$ & 4325 & 3525 & 4471 & 3517 & 7415 & 7946 & 7415 & 1.25152 \\
\hline & & & & & & & & \\
\hline
\end{tabular}

Table 1: The raw data set accompany with efficiency values 


\begin{tabular}{|c|ccccccc|}
\hline NBV & $\mathrm{w}_{1 i}$ & $\mathrm{w}_{2 i}$ & $\mathrm{w}_{3 i}$ & $\mathrm{w}_{4 i}$ & $\mathrm{w}_{5 i}$ & $\mathrm{w}_{6 i}$ & $\mathrm{w}_{7 i}$ \\
\hline$\lambda_{1}$ & -814 & 867 & 1019 & -979 & -498 & 3979 & 1309 \\
\hline$\lambda_{2}$ & -337 & 1171 & 1194 & 152 & 2805 & 2478 & 2621 \\
\hline$\lambda_{3}$ & -758 & 1657 & 540 & -1234 & 2955 & 4123 & 3719 \\
\hline$\lambda_{5}$ & 585 & 765 & 806 & 252 & -758 & 2942 & 1516 \\
\hline$\lambda_{6}$ & -126 & 342 & 212 & -1381 & 1671 & 3300 & -879 \\
\hline$\lambda_{7}$ & -256 & 1367 & 153 & -828 & 108 & 1421 & 1275 \\
\hline$\lambda_{8}$ & 505 & 1086 & 789 & 19 & 1965 & 4221 & -179 \\
\hline$\lambda_{9}$ & -814 & 423 & 627 & -505 & 413 & 4821 & 2934 \\
\hline$\lambda_{10}$ & 566 & 9 & 881 & -712 & 1384 & 3806 & 3179 \\
\hline$\lambda_{11}$ & -1186 & 1843 & 637 & 319 & -830 & 1388 & 2924 \\
\hline$\lambda_{12}$ & -591 & 608 & 936 & -258 & 2611 & 4822 & 2163 \\
\hline$\lambda_{13}$ & 674 & 854 & 856 & -1314 & 1109 & 4333 & 4222 \\
\hline$\lambda_{14}$ & 484 & 346 & 230 & 298 & 1882 & 1497 & 926 \\
\hline$\lambda_{15}$ & -357 & 1113 & 166 & -891 & -318 & 280 & 1530 \\
\hline$\lambda_{16}$ & 276 & 1763 & 874 & -812 & -1295 & 530 & 719 \\
\hline$\lambda_{17}$ & -545 & 1180 & -112 & 496 & 2781 & 2303 & -1120 \\
\hline$\lambda_{18}$ & 85 & 722 & -110 & -501 & -1577 & 3356 & -608 \\
\hline$\lambda_{19}$ & -452 & 167 & 36555 & -782 & -1335 & 3693 & 2112 \\
\hline$\lambda_{20}$ & -771 & 1512 & 541 & 414 & 1121 & 2509 & 1789 \\
\hline$\lambda_{21}$ & 174 & 1270 & 1298 & -946 & -398 & 3603 & 576 \\
\hline$\lambda_{22}$ & -903 & 575 & 894 & -1212 & 2511 & 2497 & -93 \\
\hline$\lambda_{23}$ & -452 & 1521 & 886 & 314 & 880 & 1322 & -393 \\
\hline$\lambda_{24}$ & 3589 & 1632 & 556 & -113 & 2323 & 2155 & 4300 \\
\hline$\lambda_{25}$ & 89 & 380 & 1137 & -987 & 100 & 1875 & 1406 \\
\hline
\end{tabular}

Table 2: The value of $w_{i j}$ 's in iteration 1 
the DM was asked to indicate the acceptability of the trade-offs and based on the DM's responses, a new set of weights for objective functions was obtained as $\gamma=(0.5250,0.0970,0.0001,0.1383,0.0671$, $0.0321,0.1405)$.

Iteration 2 The composite objective function was optimized which produced $\left(x_{1}, x_{2}, x_{3}, x_{4}, y_{1}, y_{2}, y_{3}\right)$ $=(3333,3720,4228,3292,5912,7324,8914)$ with $\lambda_{22}=1$ as an extreme efficient solution to the problem. The set of nonbasic variables were $\lambda_{i}, i=1, \cdots, 25, i \neq 22$ and solving model (2), by maximization of the nonbasic variable $\lambda_{i}, \quad i=1, \cdots, 25, i \neq 22$, resulted in the optimal solution $\left(x_{i 1}, x_{i 2}, x_{i 3}, x_{i 4}, y_{i 1}, y_{i 2}, y_{i 3}\right)$ with optimal value equal to 1 . Determination of efficient variables were based on the estimation of $w_{i j}$ values which were introduced in table (3).

For each nonbasic variable $\lambda_{i}, \quad i=1, \cdots, 25, i \neq 22$, model (4) were solved and variables $\lambda_{4}, \lambda_{6}, \lambda_{7}, \lambda_{9}, \lambda_{10}, \lambda_{11}, \lambda_{13}, \lambda_{14}, \lambda_{15}, \lambda_{18}, \lambda_{19}, \lambda_{21}, \lambda_{23}, \lambda_{25}$, were determined as efficient variables. Then the DM was asked to indicate the acceptability of the trade-offs. Since all the responses were no for all efficient variables, we terminated the procedure and introduced $\gamma=\left(v_{1}^{*}=0.5250, v_{2}^{*}=\right.$ $\left.0.0970, v_{3}^{*}=0.0001, v_{4}^{*}=0.1383, u_{1}^{*}=0.0671, u_{2}^{*}=0.0321, u_{3}^{*}=0.1405\right)$ as the best set of weights for inputs and outputs

Furthermore, normalization of these optimal preference common weights is associated to coefficients $\left(-v^{*}, u^{*}, w^{*}\right) \in \Re^{4} \times \Re^{3} \times 1$ of a supporting hyperplane that contains $\mathrm{T}_{v}$ in only one of the halfspaces and pass among at least one of the points of it. Therefore, we can find the value of $w^{*}$ based on the values of $v_{i}^{*}$ 's and $u_{r}^{*}$ 's accompany with the input/output values of observed DMUs. It is sufficient to solve the following model which can be performed based on simple comparisons.

$$
\begin{array}{cc}
\operatorname{Max} & w \\
\text { s.t. } & w \leq-\sum_{r=1}^{s} u_{r}^{*} y_{r j}+\sum_{i=1}^{m} v_{i}^{*} x_{i j} \quad j=1, \cdots, n
\end{array}
$$

For this example we find $w^{*}=681.736$. In this manner and due to the fact that the value of vector $\left(-v^{*}, u^{*}, w^{*}\right)$ is at hand, the output oriented efficiency score of $\mathrm{DMU}_{j}, \mathrm{j}=1, \ldots, \mathrm{n}$, can be obtained by using these common weights as $\frac{\sum_{i=1}^{m} v_{i}^{*} x_{i j}}{\sum_{r=1}^{s} u_{r}^{*} y_{r j}+w^{*}}$. These efficiency values are depicted in table 1.

\section{Conclusion}

For assessment of all the DMUs on the same scale, this paper examines the application of the Zionts-Wallenius method for generating common weights under the DEA framework. The proposed 


\begin{tabular}{|c|ccccccc|}
\hline NBV & $\mathrm{w}_{1 i}$ & $\mathrm{w}_{2 i}$ & $\mathrm{w}_{3 i}$ & $\mathrm{w}_{4 i}$ & $\mathrm{w}_{5 i}$ & $\mathrm{w}_{6 i}$ & $\mathrm{w}_{7 i}$ \\
\hline$\lambda_{1}$ & 89 & 292 & 125 & 233 & -3009 & 1482 & 1402 \\
\hline$\lambda_{2}$ & 566 & 596 & 300 & 1364 & 294 & -19 & 2714 \\
\hline$\lambda_{3}$ & 154 & 1082 & -350 & -22 & 444 & 1626 & 3812 \\
\hline$\lambda_{4}$ & 1903 & -575 & -890 & 1212 & -2511 & -2497 & 93 \\
\hline$\lambda_{5}$ & 1488 & 190 & -88 & 1464 & -3269 & 445 & 1609 \\
\hline$\lambda_{6}$ & 777 & -233 & -682 & -169 & -840 & 803 & -786 \\
\hline$\lambda_{7}$ & 647 & 792 & -741 & 384 & -2403 & -1076 & 1368 \\
\hline$\lambda_{8}$ & 1408 & 511 & -105 & 1231 & -546 & 1724 & -86 \\
\hline$\lambda_{9}$ & 89 & -152 & -267 & 707 & -2098 & 2324 & 3027 \\
\hline$\lambda_{10}$ & 1469 & -566 & -13 & 500 & -1127 & 1309 & 3272 \\
\hline$\lambda_{11}$ & -283 & 1268 & -257 & 1531 & -3341 & -1109 & 3017 \\
\hline$\lambda_{12}$ & 312 & 33 & 42 & 927 & 100 & 2325 & 2256 \\
\hline$\lambda_{13}$ & 1577 & 279 & -38 & -102 & 1402 & 1836 & 4315 \\
\hline$\lambda_{14}$ & 1387 & -229 & -664 & 1510 & -629 & -1000 & 1019 \\
\hline$\lambda_{15}$ & 546 & 538 & -728 & 321 & -2829 & -2217 & 1623 \\
\hline$\lambda_{16}$ & 1179 & 1188 & -29 & 1708 & -3806 & -1967 & 812 \\
\hline$\lambda_{17}$ & 358 & 605 & -1006 & 711 & 270 & -194 & -1027 \\
\hline$\lambda_{18}$ & 988 & 147 & -1004 & 430 & -4088 & 859 & -515 \\
\hline$\lambda_{19}$ & 451 & -408 & -239 & 1626 & -3846 & 1196 & 2205 \\
\hline$\lambda_{20}$ & 132 & 937 & -350 & 316 & -1390 & 12 & 1882 \\
\hline$\lambda_{21}$ & 1077 & 695 & 408 & 266 & -2909 & 1106 & 669 \\
\hline$\lambda_{23}$ & 451 & 946 & -8 & 1526 & -1631 & -1175 & -300 \\
\hline$\lambda_{24}$ & 1492 & 1057 & -338 & 1099 & -188 & -342 & 4393 \\
\hline$\lambda_{25}$ & 992 & -195 & 243 & 225 & -1503 & -622 & 1499 \\
\hline & & & & & & & \\
\hline
\end{tabular}

Table 3: The value of $w_{i j}$ 's in iteration 2 
approach is based on solution of an MOLP model which is intellectually consistent with the DEA philosophy. Meanwhile, the Zionts-Wallenius method does not require explicit knowledge of the DM's utility function, but uses it on an interactive basis with the DM by asking certain 'yes' or 'no' questions.

There are other methods in the literature which are also able to generate common weights. None of them are suitable to measure the preferences of a decision maker, and most of them are based on the solution of nonlinear problems. Hence, because of interactively solution of an MOLP problem that incorporates preference structures of a decision maker about input/output factors, use of our approach has an advantage over the general approaches in the literature.

When the weights of the input/output factors are available, efficiency scores can be measured. Moreover, all the DMUs can be ranked in terms of a common base. Finally, the proposed method, simply and with appropriate modifications, can be generalized to the other DEA models.

\section{References}

Belton V and Vickers SP (1993). Demystifying DEA-A visual inreractive approach based on multiple criteria analysis. Journal of the Operational Research Society 44: 883-896.

Bouyssou D (1999). Using DEA as a tool for MCDM: some remarks. Journal of the Operational Research Society 50(9): 974-978.

Charnes A, Cooper WW and Rhodes E (1978). Measuring the efficiency of decision making units. European Journal of Operational Research 2: 429-444.

Doyle JR and Green RH (1994). Efficiency and cross-efficiency in DEA: derivatives, meanings and uses. Journal of the Operational Research Society 45: 567-578.

Estellita Lins MP, Angulo Meza L and Moreira da Silva AC (2004). A multi-objective approach to determine alternative targets in data envelopment analysis. Journal of the Operational Research Society 55: 1090-1101.

Giokas D (1997). The use of goal programming and data envelopment analysis for estimating efficient marginal costs of outputs. Journal of the Operational Research Society 48(3): 319-323.

Golany B (1988). An interactive MOLP procedure for the extension of DEA to effectiveness analysis. Journal of the Operational Research Society 39(8): 725-734. 
Jahanshahloo GR, Memariani A, Lotfi FH and Rezai HZ (2005). A note on some of DEA models and finding efficiency and complete ranking using common set of weights. Applied Mathematics and Computation 166: 265-281.

Joro T, Korhonen P and Wallenius J (1998). Structural comparison of data envelopment analysis and multiple objective linear programming. Management Science 44: 962-970.

Kao C and Hung HT (2005). Data envelopment analysis with common weights: the compromise solution approach. Journal of the Operational Research Society 56: 1196-1203.

Karsak EE and Ahiska SS (2005). Practical common weight multi-criteria decision-making approach with an improved discriminating power for technology selection. International Journal of Production Research 43(8): 1537-1554.

Roll Y, Cook WD and Golany B (1991). Controlling factor weights in data envelopment analysis. IIE Transactions 23(1): 2-9.

Roll Y and Golany B (1993). Alternate methods of treating factor weights in DEA. Omega 21(1): 99-109.

Stewart TJ (1996). Relationships between data envelopment analysis and multicriteria decisionanalysis. Journal of the Operational Research Society 47(5): 654-665.

Xiao Bai L and Reeves GR (1999). A multiple criteria approach to data envelopment analysis. European Journal of Operational Research 115: 507-517.

Zhu J (1996). Data Envelopment Analysis with Preference Structure. Journal of the Operational Research Society 47: 136-150.

Zionts S and Wallenius J (1976). An Interactive Programming Method for Solving the Multiple Criteria Problem. Management Science 22(6): 652-663.

Zohrehbandian M, Makui A and Alinezhad A (2010). A compromise solution approach for finding common weights in DEA: An improvement to Kao and Hung's approach. Journal of the Operational Research Society 61: 604-610. 